Voix et Images

volxetimages

\title{
Un livre à venir. Rencontre avec Nicole Brossard
}

\section{Michel van Schendel et Jean Fisette}

Volume 3, numéro 1, septembre 1977

Nicole Brossard

URI : https://id.erudit.org/iderudit/200084ar

DOI : https://doi.org/10.7202/200084ar

Aller au sommaire du numéro

\section{Éditeur(s)}

Les Presses de l'Université du Québec

\section{ISSN}

0318-9201 (imprimé)

1705-933X (numérique)

Découvrir la revue

\section{Citer ce document}

van Schendel, M. \& Fisette, J. (1977). Un livre à venir. Rencontre avec Nicole Brossard. Voix et Images, 3(1), 3-18. https://doi.org/10.7202/200084ar d'utilisation que vous pouvez consulter en ligne.

https://apropos.erudit.org/fr/usagers/politique-dutilisation/ 


\section{Un livre à venir}

Rencontre avec Nicole Brossard

Michel van Schendel et moi-même avons rencontré Nicole Brossard à deux reprises dans l'intention de l'interroger sur les livres passés, sur ce que signifient pour elle les notions de littérature, roman, poésie, écriture, texte, etc. Mais plutôt qu'une entrevue, c'est une discussion qui s'est établie où chacun à son tour était questionné. Et, ce n'est qu'à la fin de la seconde rencontre qu'on a découvert qu'en fait, on n'avait parlé que du livre que Nicole Brossard s'apprête à écrire: I'Amer ou le chapitre effrité à paraître aux éditions Quinze. (Jean Fisette)

\section{Les pratiques d'écriture}

J. F. Sous la rubrique du «roman», tu nous as donné Un livre ${ }^{1}$, Sold-out ${ }^{2}$ et French Kiss ${ }^{3}$; après la poésie qui, somme toute, était assez peu figurative, cette entreprise était surprenante, si l'on songe à ce qui s'appelle habituellement "roman». Alors, je te demande à quel besoin correspondait cette entreprise?

M. v. S. Moi, je ne suis pas d'accord avec cette opposition poésie/roman; la poésie de Nicole Brossard me semble au contraire très figurative: l'identité corps-texte par exemple réalise le comble du figuratif.

N. B. J'ai écrit Un livre après Suite logique ${ }^{4}$ et le Centre blanc ${ }^{5}$; je tiens à le rappeler car il y a là un lien très important. Lorsque j'écrivais le Centre blanc, je croyais qu'avec la poésie, on pouvait aller très loin: dépasser le silence et tout dire. Mais j'ai découvert que l'essentiel, c'est-à-dire l'extrême douleur et l'extrême plaisir, on ne pouvait le dire consciemment avec des mots. Puis, il y a eu lin temps de silence où dominait le sentiment de faillite à l'égard de ce que j'avais investi dans l'idée même de poésie. J'ai alors beaucoup lu et très peu écrit; j'en suis arrivée à croire qu'on ne peut dire le plaisir que si l'on a du plaisir en écrivant. C'est peut-être aussi simple que ça: écrire que ce n'est pas simple. Puis je suis arrivée au roman : le roman, c'est un prétexte - sans jeu de mots - qui utilise aussi les personnages comme prétextes. Dans Un livre, - il faut le distinguer des deux romans suivants - je porte un regard sur moi-même en train d'écrire; c'est très clair en général, il y a 
deux paragraphes dans chaque page; dans le premier, un tableau constitué de peu d'événements, peu de sentiments et des personnages à peine esquissés sur le plan psychologique. Dans le second paragraphe, c'est moi en train d'écrire. Dans les deux autres livres, et surtout dans French Kiss, le même dédoublement se réalise sur le plan du signifiant et non plus du signifié. Dans Un livre, le procédé était assez simple tandis que dans French Kiss le procédé est à peine suggéré puisqu'il entre dans le jeu même de l'écriture. Par exemple, le rouge et le noir, les poils raides et noirs, etc. C'est que je ne dis pas ce que je vois, ni ce que j'ai pensé en écrivant, je ne fais aucune démonstration.

J. F. Je reviens à Un livre: tu parlais d'une dissociation entre les deux perspectives réalisées dans les deux paragraphes de la même page: n'y a-t-il pas là une scission qui révélerait un sur-moi analytique puissant qui cacherait une gêne à se laisser emporter dans la fiction, une sorte de résistance?

N. B. Oui, c'est une résistance et une conscience de cette résistance. Parce que je sais qu'écrire des livres, au fond, c'est toujours une façon de survivre, c'est toujours un prétexte pour continuer d'avoir l'air d'aller dans l'existence. Et je remets toujours en question ce que je suis en train de faire. C'est pour cela que mes manuscrits se corrigent au moment même où je les écris. C'est très rare que je corrige par la suite. Peux-tu revenir sur ta question?

J. F. Je t'interrogeais sur la signification de la scission et puis s'il n'y avait pas une gêne à se laisser aller dans la fiction, à s'abandonner.

N. B. Ce n'est pas une gêne dans le sens de pudeur, mais une gêne dans le sens du rapport conscient/inconscient que j'essaie toujours de projeter dans un inconscient social. C'est-à-dire, non pas l'inconscient de ma propre histoire, mais l'inconscient de mon conditionnement social. Une image m'arrive et je l'écris; mais presque immédiatement, j'y reviens parce que j'aurai pris conscience qu'elle fait état d'un conditionnement social que je refuse et automatiquement j'essaie de renverser la roue. C'est pourquoi, pendant que j'écris, je jette toujours un coup d'œil sur ce qui vient de s'écrire, ou peut-être est-ce simultané.

J. F. Alors la scission dont je parlais - disons, pour simplifier entre narré et analyse - correspond à une dialectique qui concerne ton rapport aux choses, au monde, à la société: il y a le conditionnement social et l'anticonditionnement de ta réaction. C'est donc dire que de ta poésie à ton roman, il y a eu rupture dans cette volonté de prendre conscience des choses qui s'est manifestée et qui fait partie du texte, de l'écriture?

N. B. Oui. Les premiers recueils, je les ai écrits dans le plaisir, mais d'une façon très inconsciente, je crois; et j'ai beaucoup de difficulté à repérer l'inconscient sous-jacent à ces textes-là. Alors que dans les romans, dans la Partie pour le tout ${ }^{6}$, puis dans certains autres textes qui ont une grande importance pour moi, «E muet mutant" 7 et "le Cortex exubé- 
rant $^{8}$ " surtout, je peux repérer facilement ce qui se passait chez moi au moment où je les ai écrits. C'est que je suis maintenant beaucoup plus près de mes textes que je ne l'étais auparavant. Ce qui crée une autre difficulté, c'est le risque de faire surgir l'urgence et d'évacuer le plaisir. $\mathrm{C} a$, c'est une inquiétude que j'ai eue il y a un mois. Mais maintenant que je vais commencer à écrire, je pense que je ne peux faire autrement que d'opter pour la dimension de l'écriture en noyant l'urgence, en écrivant l'urgence, ce qui va probablement ressortir à la lecture; je veux noyer l'urgence dans le plaisir du texte. Parce que si je ne le fais pas à ce moment-là, je ne vois pas en quoi la démarche que j'aurai suivie pourrait s'inscrire dans une écriture.

M. v. S. Qu'est-ce que tu t'apprêtes à écrire?

N. B. C'est une fiction théorique. Je sens qu'il y a des choses que je vais devoir m'expliquer clairement; mais en même temps, elles seront sans cesse impliquées dans des fantasmes, des rapports symboliques, des jeux de mises en scène, etc.

M. v. S. Est-ce que cette fiction théorique vient du roman au sens où tu viens d'en parler? Et si c'est le cas, est-ce que l'écriture, comme celle du roman, serait une écriture non pas simplement de la conscience de la conscience de quoi? - mais une écriture de la conscience de la rupture entre le plaisir immédiat et le sentiment de la place à occuper ou que l'on occupe effectivement - en ce qui concerne la socialisation?

N. B. Dès que l'on utilise ce que l'on appelle la prose, c'est-à-dire dès que l'on dépasse l'instant, la synthèse - la poésie, c'est l'ellipse par excellence - dès que l'on veut relier l'instant présent à celui qui l'a précédé, on est pris dans une histoire. Alors, on se situe par rapport à une dialectique entre le conditionnement, le social qui intervient sans cesse et le plaisir, la jouissance.

M. v. S. Qui intervient comment ? Pour l'épouser, pour être à l'unisson du plaisir ou bien au contraire pour le réprimer?

N. B. ... qui intervient pour le réprimer. Alors, si dans un roman, j'ai plus de souffle pour m'inscrire dans une histoire, dans un passé, dans un présent, la lutte au fond, se fait toujours entre un conditionnement passé qui tente de s'inscrire sur la page et en même temps un plaisir de l'inscription sur la page qui vient comme pour rejeter tout le conditionnement exercé sur moi par la société dans laquelle je m'inscris.

J. F. Alors, est-ce que la pratique d'écriture n'apparaîtrait pas comme un geste qui aurait comme fonction d'établir une solution de continuité dépassant l'opposition dont on parlait au début entre le plaisir personnel et la répression du social?

N. B. C'est effectivement une continuité: dans la mesure où je suis un être social, je fonctionne entourée, je fonctionne dans une hiérarchie et même si je m'y refuse, elle demeure et je ne puis en faire abstraction; 
écrire, c'est tenter une continuité plausible d'existence dans laquelle puisse s'inscrire un plaisir qui a ceci d'exceptionnel qu'il est productif.

J. F. Alors ta pratique d'écriture deviendrait, d'une façon plus générale, le modèle d'une façon de vivre au-delà de l'opposition plaisir - répression?

N. B. Elle est une façon de vivre, un apprentissage aux mots et aux choses de la vie. Elle ne devient pas modèle parce que déjà le modèle serait quelque chose à suivre, donc par le fait même, potentiellement répressif.

M. v. S. Je reviens tout de même à la première partie de ma question: la fiction théorique que tu t'apprêtes à écrire, est-ce qu'elle participe à ce que l'on a tenté de cerner comme étant, selon toi, l'écriture romanesque? Est-ce que ça participe de cette conscience de la rupture?

N. B. Ça participe de la conscience de la rupture, oui. Ça l'élargit même dans ce sens qu'il y a toute une dimension dans ce livre que je ne connais pas encore, mais que je sens très différente des autres dimensions qui se sont inscrites dans les premiers romans.

M. v. S. Donc, il y a un élargissement du nombre de registres, une multiplication des niveaux d'écriture?

N. B. Oui.

M. v. S. Le roman c'était la première expérience de cette multiplicité des registres de l'écriture?

N. B. Oui.

M. v. S. Là, ça se déploie?

N. B. Oui, c'est ça. Dans la poésie, ce type d'écriture n'existait pas. Au fond, il faut être très innocent pour écrire des poèmes. II faut déjà s'être enfermé.

M. v. S. Une innocence très perverse ou très rusée, je crois!

N. B. Peut-être les deux. Mais je pense que pour écrire de la poésie, il faut être assez innocent dans ce sens que l'on se tient à distance du pouvoir. II n'y a pas de volonté d'inscrire du pouvoir sous une forme connue ou éventuelle. Écrire de la poésie, c'est travailler dans l'abîme. On a été stimulé par l'extérieur et c'est sans doute pour cette raison que l'on réagit en écrivant des poèmes. Mais on n'a pas répondu à l'extérieur. Tandis que dans le roman, on se sent concerné au point que l'on tente de donner la réplique. C'est justement ce qui fait cette rupture entre le plaisir - où l'on est seul - et la répression. Écrivant de la poésie, on se dit: la répression est là et je suis bien dans mon plaisir. Alors il y a tout un vécu qui se fait au présent, sans hiérarchie, sans lutte si je puis dire, sinon celle liée à toute forme d'exploration. 
M. v. S. Tu crois que dans ce que tu appelles l'écriture poétique, il n'y a pas de répression et, par conséquent, pas de luttes défensives ou offensives contre la répression. Est-ce là ce que tu appelles l'innocence?

N. B. Oui, c'est-à-dire que s'il y a une lutte qui surgit, elle ne tend pas à convaincre ou elle n'appelle pas de réponse. II n'y a pas de conversation ni de dialogue dans la poésie, c'est un tout que l'on prend tel quel. Tandis que dans le roman, il y a toujours "je vous considère", "je vous réponds", "vous me considérez", «je vous réponds», il y a un rapport continuel et dynamique. Dans la poésie, ce rapport, je le perçois comme intensité plutôt que comme dualité.

J. F. Mais finalement, les termes de poésie et de roman n'ont plus de sens?

M. v. S. Justement, est-ce que tu ne sanctionnes pas la distinction des genres?

N. B. II y a des distinctions formelles entre poésie et roman. Une technique différente pour apprivoiser ou exorciser les choses de la vie. Si je dis que je fais fi des genres, c'est en ce sens que je suis mon propre rythme et mes besoins quand j'écris. Pour parler des besoins que je sens présentement en termes d'écriture, je choisis l'expression "fiction théorique", ce qui pourrait englober tout à la fois le romanesque, l'analyse et la poésie.

M. v. S. Est-ce que ce n'est pas tout ça le discours poétique? Et ici, je pense surtout à la multiplicité des registres et par conséquent, la conscience et la pratique de l'écart entre ces registres; dans leur conjonction simultanée, il y a une énorme contradiction qui est proprement poétique.

N. B. Cette conjonction simultanée, je la perçois quand j'analyse deux moments de ma vie: une étape d'innocence où, si j'étais en lutte, je l'étais isolément comme tout individu qui tente de survivre dans une société donnée. Maintenant j'ai le sentiment d'être en lutte à la fois isolément et à l'intérieur d'une solidarité. C'est ce qui me ramène au rapport dynamique dont je parlais tantôt.

\section{Le plaisir, la jouissance}

J. F. Je me permets ici de prendre une voie parallèle. Tu as fait un très beau texte intitulé: “le Cortex exubérant ". Cette association du corps et du texte - d'ailleurs omniprésente chez toi - ne correspond-elle pas à un pont jeté entre deux positions apparemment irréductibles?

M. v. S. Cette association corps/texte est présente sur le plan thématique, sinon sur celui du signifiant, depuis le premier recueil Mordre en sa chair $^{9}$. Je prends ce passage:

Pendant ce temps les yeux collent à la peau lumière coupure 
on étire le ventre de l'angoisse

la vague croissante des brumes.

On arrête les horizons

au bout des vertiges.

Tout se brise

l'éclat lance au zénith

des arbres à fleur de bronze

pendant ce temps mon cœur vertige

sur toison de soleil.

L'alliance de l'angoisse, des yeux qui collent à la peau, de la vague, est-ce que ce n'est pas déjà, sur le plan thématique, l'annonce de ce qui va traverser à peu près tout ce que tu as écrit jusqu'à la Partie pour le tout où la même association fonctionne de façon très nette sur le plan du signifiant. Par exemple, à la page 27 , je lis: “... un lapsus/me mouille... " Le rapport langue / ventre est marqué par un lapsus qui n'est énoncé comme lapsus que par l'intermédiaire de l'organe langue. D'ailleurs, dans le langage populaire, on dit: «la langue me fourche». Cette ambivalence repose sur les deux sens du mot "langue" assumés simultanément. Ce déplacement - ou cette continuité métonymique - conduit à la langue comme organe sexuel. Alors là, le rapport d'identité corps / texte est très nettement marqué. Mais est-ce que depuis le début, il n'y avait pas cette ambition-là?

N. B. Je pense que c'est là depuis le début et que tout se concrétise, particulièrement dans French Kiss qui est vraiment l'illustration de ce que tu dis. French Kiss, c'est l'histoire d'un baiser et ça, c'est l'histoire de la langue employée dans les deux sens. C'est l'exploration du corps de l'autre avec sa langue «linguistique» et organique et c'est à la fois l'exploration de sa propre bouche avec sa propre langue. L'exploration que j'ai faite dans ce texte, je l'ai trouvée absolument passionnante parce que j'avais une glissoire qui me permettait de dédoubler, de revenir à un seul sens, de dédoubler de nouveau, de tripler, de quadrupler tant que c'était possible. C'est un jeu auquel je me suis prêtée avec le titre par exemple.

M. v. S. Mais est-ce qu'il ne subsiste pas une ambiguïté? Tu poses les deux sens de langue, soit la langue comme organe et la parole. Mais de la parole au texte, il y a une distance qui, me semble-t-il, empêche l'identité corps/texte.

N. B. Le jeu de mots est là, au départ, dans ma tête donc il est déjà dans ma bouche; je peux l'articuler, le prononcer; en d'autres termes, il est sans surprise s'il est dans la parole. Mais, dès qu'il s'inscrit dans un texte, il devient plein de surprises et c'est cela qui fait l'écriture.

M. v. S. Oui. Donc il n'y a plus d'identité à ce moment-là. II n'y a plus d'identité entre le corps et le texte si le texte, c'est de l'écriture. Alors corps-texte, ce n'est plus, au bout de la ligne, qu'une métaphore?

N. B. Bien oui, parce que les mots ne seront jamais le corps. 
M. v. S. Ah! Voilà ce que j'essayais de te faire dire.

J. F. C'est reconnaître la limite de l'association corps/texte.

M. v. S. Et de l'identité.

N. B. La limite on ne peut la connaître. Comme je le disais tantôt, je pourrai écrire le restant de mes jours parce que je ne connais pas la limite.

M. v. S. D'accord! Mais qu'est-ce qui te permet de penser l'identité qui est établie comme telle dans ton texte?

N. B. C'est la recherche de l'unité. C'est la sortie de la dialectique. C'est la recherche de l'extase. C'est la recherche de l'unité où il n'y. a plus de besoin, où il n'y a plus de dépendance. Où il n'y a plus de rapport en fait. C'est l'instant, c'est le présent.

J.F. Cette perspective dans laquelle tu te places, c'est théologique?

N. B. Oui ça rejoint le théologique; quand on parle de l'extase, ça rejoint une forme de mysticisme, c'est l'unité dont je parlais.

J.F. Avec le terme de jouissance comme point d'aboutissement d'une téléologie. C'est donc qu'il y a quête?

N. B. Point ultime, certainement mais je ne vois pas pourquoi tu dis cela?

J.F. C'est tout simplement une remarque que je me fais. Je me demande si l'on ne rejoint pas là des schèmes qu'on a voulu rejeter à une certaine époque de nos vies et qu'on retrouve toujours?

N. B. Oui, mais pas tout à fait. Tu fais référence à la religion dans laquelle on a passé notre enfance. Je crois qu'il y a de l'ésotérisme, du mysticisme dans ce point oméga. Depuis la religion de notre enfance, il y a eu des concrétisations, des matérialisations de ces fantasmes qui, de toute façon, existaient depuis toujours. Les religions ont pu être codifiées, ça n'a rien à voir avec la matérialisation, par exemple, de ce fantasme qui est là depuis des centaines d'années, le corps-texte.

Moi, je suis un être de désir, donc un être de mots, un être qui cherche son corps et cherche le corps de l'autre: pour moi, c'est toute l'histoire de l'écriture.

M. v. S. L'écriture comme désir et non pas comme plais̀ir.

N. B. L'écriture étant le plaisir.

J. F. Le désir tendu vers le plaisir.

M. v. S. C'est la tendance au plaisir. C'est la recherche du plaisir, mais jamais atteint.

N. B. C'est cela.

J. F. Et c'est dans la mesure où le plaisir ne sera jamais pleinement satisfaisant qu'il y aura toujours place pour l'écriture. 
N. B. Effectivement. Dans les premiers recueils, je croyais naïvement pouvoir dire l'essentiel du corps. Après le Centre blanc j'ai compris qu'il n'y aurait jamais identité entre le corps et le texte. Puis, après avoir connu les désolations et les risques de la répétition, j'ai reconnu qu'à l'usage des mots, tout devenait possible. C'était l'éventail.

J.F. Mais tout reste à faire en même temps?

N. B. Tout reste à faire en même temps en fonction de mon propre désir, celui-ci se modifiant sans cesse.

M. v. S. L'identité, puisqu'elle est celle du plaisir et que le plaisir n'est jamais atteint que dans la recherche du plaisir, n'est donc jamais atteinte. Le plaisir est à la fois atteint et non atteint par l'écriture en tout cas.

N. B. Oui c'est cela exactement. Tout ce que l'on peut faire quand on écrit, c'est d'inscrire ou tenter d'inscrire comment pourrait se produire le plaisir, non pas le plaisir, mais plutôt l'extase ou la jouissance. C'est Barthes qui fait cette distinction entre le plaisir et la jouissance et je la trouve très pertinente: la jouissance est asociale et le plaisir, étant social, est lié à des rapports que l'on entretient avec les autres, rapports de puissance, dépendance, etc. La jouissance ne peut s'inscrire dans l'acte même d'écrire parce que l'écriture étant une production, est possiblement récupérable. Alors que la jouissance est totalement irrécupérable par qui que ce soit. Ça se vit, ça ne se donne pas, ça ne se prend pas.

M. v. S. Oui, parce que c'est la grande dépense. C'est Bataille ça. Effectivement il n'y a pas d'accumulation.

\section{Du langage de l'action au désarrol}

J. F. Cette écriture qui produit le plaisir et à la fois qui provient du plaisir, est-ce que ça définirait ce que l'on appelle l'écriture féminine, en la différenciant d'une écriture qui reproduit un discours idéologique, un discours du pouvoir?

N. B. Non je ne pense pas. Et je le dis par rapport à ma propre expérience d'écriture que je considère comme étant celle d'un écrivain. L'écriture repose sur tout un circuit qui est actuellement monopolisé par les hommes; c'est-à-dire que les hommes ont plus facilement un rapport à l'écriture que les femmes en général. Ce sont des conditionnements qui font que les hommes sont plus susceptibles de s'affirmer comme sujets que les femmes. Or écrire c'est, selon moi, s'affirmer comme sujet. Et mon rapport à l'écriture est un rapport de sujet, tandis que pour la majorité des femmes, le rapport en est un d'objet.

M. v. S. C'est également vrai au Québec que les femmes ont moins que les hommes la puissance d'accéder à l'écriture? 
N. B. Je pense que c'est aussi vrai au Québec. Évidemment beaucoup de femmes ont écrit des livres, et des livres qui sont marquants dans l'histoire de notre littérature. Ça n'efface pas le fait qu'en général, les femmes sont perçues comme objets dans notre société. Ce phénomène rend tout simplement plus évident le fait qu'au Québec, les femmes vivent avec des hommes qui sont eux-mêmes sans pouvoir politique ni économique, avec un piètre pouvoir culturel. II faudrait aussi tenir compte des faits historiques qui expliquent très partiellement ce phénomène: ici la plus grande scolarisation des femmes.

J. F. Mais tu n'as pas répondu à ma question: comment se situe l'écriture féminine par rapport à la dialectique du système répressif et de la quête du plaisir?

N. B. Toute écriture pour moi s'inscrit dans une expérience du plaisir, quelle que soit sa médiocrité, parce que c'est un acte de déconditionnement, de décolonisation que l'on opère par rapport à soi en tant que sujet.

M. v. S. Donc à ce moment-là, c'est un rapport à la jouissance?

N. B. L'écriture c'est un acte qui s'inscrit dans le plaisir parce que c'est un acte gratifiant. C'est un acte de sublimation; ce n'est pas un acte de désespoir, ce n'est pas un acte suicidaire, c'est au contraire un acte de vie. Quand on emploie l'expression “écriture féminine», ça permet aux écrivains, aux hommes en général, aux professeurs, de se situer en fonction d'une expression qui n'est pas d'eux. Ça inverse totalement l'attention que l'on porte au texte écrit par une femme. Si les femmes n'attirent pas elles-mêmes l'attention sur leur texte, avec leur propre expression, alors il y a risque que l'on dise: "encore un texte de fernme». Ça, c'était une parenthèse sur l'expression «écriture féminine». Si l'on continue dans cette veine-là, on va pouvoir parler d'un contenu, d'une expérience de vie qui jusqu'à maintenant a été peu dite ou racontée dans les textes; ou si elle l'a été, elle l'a été toujours d'une manière biaisée ou en tout cas n'a jamais acquis une crédibilité en tant que telle.

M. v. S. Lors de notre dernière rencontre, tu nous disais qu'au fond, l'écriture féminine ça n'existe pas pour toi.

N. B. J'aime employer l'expression, mais en termes stratégiques. Parce qu'en fait le rapport qu'un sujet a à l'écriture à mon avis est identique pour un homme et pour une femme. Ce qui est important, c'est la notion de sujet. C'est un rapport qu'un sujet pose.

J. F. Et est-ce que le rapport à l'écriture ne reproduit pas le rapport aux choses? Si l'on pose que l'homme et la femme voient les choses de façons différentes, il s'établirait nécessairement une différence dans le rapport à l'écriture.

N. B. Je pense que les femmes qui entrent dans une production, qu'elle soit de l'ordre de l'écriture ou qu'elle soit de l'ordre de l'architecture ou 
de la finance, entrent dans une production. Et qu'entrer dans une production suppose des comportements qui, jusqu'à maintenant, ont été qualifiés de masculins. Mais, dans les faits, le rapport est toujours le même. Là où le rapport à l'écriture est différent, c'est quand on commence à écrire, à se penser comme sujet. Alors il y a toute une démarche d'hésitation, de peur, de crainte, d'avance, de retrait qui font que cette production se rapproche du journal. II y a parfois du silence, du mutisme, l'oppression ça laisse des traces. La grande différence entre hommes et femmes dans le rapport à l'écriture, c'est que les femmes ont peur d'écrire, comme on dit avoir peur de prendre l'initiative, c'est ça la différence. C'est ça qui me fait mal. De voir à quel point, nous les femmes, avons été mutilées, empêchées d'une vision du monde. Écrire c'est aussi ça: avoir une vision.

M. v. S. Et le journal, c'est le journal quotidien ou le journal littéraire?

N. B. Le journal comme genre, littéraire.

J.F. Et le journal, c'est le prototype même de l'écriture où le sujet se donne comme objet, puisque dans le journal, on parle de soi. Le journal, c'est le je-moi.

N. B. Oui, c'est très juste.

M. v. S. C'est le je-moi qui fait la dispense du ça.

J.F. Et le ça, c'est le pouvoir. Alors le journal est une pratique narcissique?

N. B. J'ai remarqué dans la nouvelle production des textes faits par des femmes, que la tendance était à produire des textes qui relèvent du journal intime. Et moi-même, dans une période de questionnement, je me suis presque trouvée à produire ce type de texte. Et c'est normal.

J. F. Comme les six monologues de la Nef des sorcières ${ }^{10}$; ces six monologues sont construits sur le schème du journal personnel.

N. B. Comment les monologues opèrent, c'est autre chose. Parce que les monologues de la Nef opèrent dans la mesure où c'est nous qui avons la place, c'est nous qui formulons notre propre négociation avec le public. De fait, c'est le public au théâtre qui est en attente, et le théâtre offre une scène qui, pour des raisons culturelles, jouit d'une crédibilité. On aurait pu se raconter ça ici dans le salon, il n'y aurait pas eu de crédibilité, de la part des hommes en tout cas.

J.F. Est-ce que l'entreprise de la Nef des sorcières, j'y reviens, qui était fondée sur la forme du monologue, et précisément celui du journal intime, n'avait pas pour fonction de pousser à bout cette expérience de l'écriture autobiographique ou narcissique et de pouvoir dire: maintenant on est allé au paroxysme, c'est fini l'écriture en secret devant le miroir dans le coin de sa chambre? 
N. B. On ne peut pas dire que c'était de mettre un point final; certaines femmes seulement se rendent compte que pour elles, c'est fini ce type de monologue. Mais il s'agit là d'une expérience fondamentale: c'est l'inscription de l'objet devenant sujet.

J. F. Alors s'il y a une transgression dans la Nef des sorcières, c'est d'avoir rendu public le journal intime?

N. B. Oui et il y a transgression de l'habitude aussi dans la mesure où le contenu, s'il était familier, n'avait jamais été accrédité. Et aussi d'inscrire le drame entre la scène et les spectateurs plutôt que de le situer uniquement sur scène.

M. v. S. Est-ce qu'on ne pourrait pas ajouter qu'il s'agit, de la part de la femme, d'une appropriation du sur-moi qui traditionnellement a toujours été dévolu à l'homme, sauf que l'homme ne se soit jamais manifestement approprié ce sur-moi, sur une scène publique? En disant moi-je, on fait abstraction, non du tu auquel on continue toujours de s'adresser, mais du il; cette troisième personne, qui est d'ailleurs toujours au masculin - on ne dit pas "elle" - il ne s'agit de la reconnaître que dans la mesure où on peut la conquérir et même l'écraser pour lui substituer le moi-je féminin.

N. B. D'abord, je fais une distinction entre le je et le moi-je.

M. v. S. Oui, entre le sujet et le moi-je qui est un sujet redondant.

N. B. C'est juste; on dit familièrement: nous autres. Cette expression indique qu'on ne sait pas qui on est et elle tente de renforcer une connaissance de soi; mais, en fait, l'expression "nous autres" révèle une distance: “Nous autres, les Québécois", “Nous autres, les femmes", etc. En général, ce sont toujours les colonisés qui emploient ce renforcement.

M. v. S. Mais il me semble que tu lui as tout de même donné raison lorsqu'il disait du journal intime que c'est le discours du moi-je?

N. B. Je lui ai donné raison parce que, par la suite, il a parlé d'objet. Ce que je voulais dire, c'est qu'au fond, dans l'expérience du journal intime devenu livre, dans le roman par exemple, l'objet apprend à devenir sujet et c'est l'expérience de ce premier livre qui en général mène à l'écriture et au plaisir du texte. Pour beaucoup de femmes, il faut passer par l'expérience du journal pour apprendre à connaître le moi-je, parce qu'il n'a jamais été réalisé.

M. v. S. Et le moi-je donne «nous autres "; c'est un rapport de dissociation.

N. B. En fait, c'est juste. Dans les textes de la Plaque tournante ${ }^{11}$, c'est de tout ça dont il est question. C'était un texte de commande sur la femme et l'écriture: j'essayais de me voir en tant que femme et de trouver là la spécificité de mon conditionnement de femme dans l'écriture; j'étais 
dans le désarroi le plus complet parce que habituellement, quand je prends une plume et une feuille de papier, c'est l'écrivain avec tous ses rapports à l'écriture qui se manifeste. En général, mon rapport à l'écriture est de l'ordre du plaisir; là il était devenu un rapport de culpabilité parce que le plaisir est difficile à vivre quand la majorité des femmes ne l'ont pas eu et que tu comprends ta solidarité avec elles. Toutes les chaînes de la culpabilité se sont mises à fonctionner et il y avait un déchirement perpétuel; j'ai commencé à écrire «je»; par la suite j'ai écrit moi-je et j'ai commencé à réfléchir sur ce problème: je ne comprenais pas, je ne pouvais plus écrire «je». Ce même désarroi, je l'avais connu en écrivant le texte de la Nef des sorcières. J'étais incapable d'écrire; alors j'ai rencontré la comédienne qui m'a dit: «Écoute! Fais comme tu fais toujours quand tu écris"; et c'est comme ça que le texte s'est amorcé avec des abat-jour et des ombres chinoises, des choses qui n'ont rien à voir avec l'écrivain femme.

M. v. S. Tu dis n'utiliser l'expression d'écriture féminine que par stratégie et d'autre part, tu affirmes rechercher la spécificité d'une écriture de femme; ces deux positions sont inconciliables. Est-ce qu'il n'est pas là le désarroi : tenir cette contradiction?

N. B. Contradiction qui, je pense, s'achève avec le début de la rédaction de cette fiction théorique dont j'ai parlé. C'est cette contradiction qui a fait que pendant deux ans, je n'ai produit aucun texte de fiction sauf celui de la Nef des Sorcières et celui de la Plaque tournante, deux textes qui mont complètement déchirée quand je les ai produits.

M. v. S. Tu crois que c'est possible d'arriver à une réconciliation?

N. B. Je ne le sais pas, je n'en suis pas encore à la rédaction, mais je sens que ce sera une tentative de réconciliation de cette contradiction dans laquelle j'ai été depuis deux ans.

M. v. S. Le sujet sera moi-je?

N. B. Non. Moi-même exactement. Du début à la Partie pour le tout, il y avait le sujet; puis ces deux textes de femme écrivain avec le moi-je; maintenant, je tente la réconciliation des deux et quand je dis réconciliation, ça s'opère au niveau formel aussi.

M. v. S. Et comment vas-tu réconcilier ça?

N. B. Je ne sais vraiment pas et il est possible que ce soit l'ancien sujet qui remporte le tout; c'est-à-dire le sujet écrivant. Je n'en sais rien, mais actuellement je dispose d'une expérience de deux ans, d'une réflexion, d'une analyse, d'une vulnérabilité totalement inédites. Je présume que ça devrait s'articuler formellement d'une manière inédite.

J. F. Tu n'as aucune certitude prédéterminée. Tu te jettes dans le travail un peu comme on se jette à l'eau?

N. B. Comme dans tous les textes que j'ai écrits. 


\section{Journal d'analyse ou journal intime?}

J. F. Tu t'es relativement éloignée de la fiction depuis quelque temps. Pourquoi?

M. v. S. Ça fait du bien d'être en vacances par rapport à la fiction, non?

N. B. Parfois, mais il faut pouvoir choisir la date de ses vacances. Ce qu'en général, j'ai toujours fait.

M. v. S. Et là, tu te trouves en défaut de stratégie par rapport au choix de tes dates, de tes cycles?

N. B. En défaut d'un rythme qui a été brisé d'un coup.

M. v. S. Ça fait du bien d'avoir l'expérience de la brisure?

N. B. Absolument, et ce n'est que connaissance, toujours. C'est très drôle parce que plus la date du début de l'écriture se rapproche, plus je fais mon choix, et je me dis qu'il faut apprendre à connaître sa fonction dans la société. Moi je choisis la fonction d'écrivain avec une solidarité de femme dans la pratique verbale et journalistique.

M. v. S. Journalistique au sens du quotidien que tout le monde lit ou journalistique au sens du journal intime?

N. B. Au sens du journal que tout le monde lit; je veux dire au sens de l'analyse et de l'information.

M. v. S. Ce qui est aux antipodes du journal intime qui lui n'apporte aucune information. Ou plutôt qui apporte à celui qui l'écrit une information qu'il connaît déjà.

N. B. C'est d'une mémoire à réapprendre dont il s'agit. En le rédigeant, les gens prennent conscience de ce qu'ils ont vécu mais en même temps, c'est l'affirmation d'un refus à la fois tourné vers le passé et vers le futur.

M. v. S. D'accord et à partir de là, on rejoint la question de tout à l'heure. II s'agit du passage de la scène primitive du journal intime à la scène de la négation, à la fois de ce journal et du rapport de domination qui l'a produit; c'est là la conséquence de l'étalement du journal intime devant le grand public: à ce moment, le journal devient politique.

N. B. Ça devient effectivement un journal politique: il y a toujours ce renvoi continuel de l'intime au politique.

M. v. S. Et c'est la raison pour laquelle tantôt tu as fait ce passage en employant le mot journal, sans le spécifier?

N. B. Parce que je m'apprête à écrire dans un journal politique. Quand je pratique ce genre d'écriture, j'ai des idées que je mets les unes à la suite des autres. 
J. F. Ce n'est pas une écriture d'exploration?

N. B. Non pas du tout; c'est une écriture qui confirme une analyse, une réflexion précédente.

M. v. S. C'est cela pour toi un journal politique?

N. B. Pour moi, oui. Certaines choses s'ajoutent à mesure du travail, mais au départ, je connais déjà la conclusion.

M. v. S. C'est curieux; j'ai une assez longue expérience de journalisme et pour moi cette écriture est tout à fait différente: elle est faite d'inquiétudes et d'incertitudes. Cette écriture, c'est un cheminement: je connais le chemin à mesure que j'y marche.

N. B. Ah non! II y a une question et une réponse qui ont déjà été posées suite à une réflexion et qui ne font que se matérialiser dans ce que j'appelle « article».

M. v. S. Mais cet article que tu envisages, c'est ton article à toi: c'est encore là le moi-je qui prédomine?

N. B. Disons par exemple l'article que j'écris actuellement.

M. v. S. Tu parles de toi, là?

N. B. Je parle à partir de moi.

M. v. S. C'est très différent.

N. B. Oui, je parle de mon expérience d'écriture journalistique. Ce travail m'a toujours ennuyée alors je n'ai jamais investi beaucoup pour acquérir une discipline. Par exemple, j'écris un texte sur les belles-mères: il y a toute une analyse à faire: c'est quoi une belle-mère? pourquoi les belles-mères sont-elles si détestées? Pourquoi toutes ces blagues, etc.?

M. v. S. Peut-être tout simplement parce que c'est une généralité et que les généralités sont fausses.

N. B. C'est que la belle-mère, c'est toujours la mère de l'autre; alors on rend à la mère de l'autre, ce que l'on est impuissant à rendre à la sienne. L'article, c'est cette réponse qui était là; il ne s'agit plus que de l'expliquer. Mais qu'est-ce qui m'a amenée à cette réponse?

J. F. Ce qui est donné dans l'analyse rejoint l'inconscient?

N. B. L'analyse rejoint le conscient et les généralités tandis que le texte de fiction provient de l'inconscient, ce qui n'a pas de mots et qui pourtant prend forme. La nuit, nous ne distinguons pas les formes qui sont pourtant là si vives au matin.

J. F. C'est intéressant en ce sens qu'on arrive à établir une sorte de typologie des textes chez toi: d'abord le premier texte elliptique, intuitif, statique; puis l'analyse et sa reproduction pure et simple dans l'article et, enfin, le texte de fiction comme French Kiss, Sold out ou celui que tu 
entreprends qui est double: induction et analyse qui permet de pousser plus loin l'induction, et ainsi sans fin...

M. v. S. II y a donc une continuité entre ta propre biographie et ta bibliographie d'écrivain: l'écriture poétique, l'écriture dite romanesque, expérience déjà d'une multiplication de registres et le livre que tu prépares. Nous ne parlons que de fiction théorique depuis deux heures: c'est une continuité ça?

N. B. Il y a continuité certainement. J'ai intitulé un recueil Suite logique pour inscrire le rapport de ma propre expérience du texte et de la vie. II ne peut pas ne pas y avoir de suite logique.

M. v. S. Je ne sais pas s'il y a une suite logique par rapport à la vie, mais il y a certainement une suite logique par rapport au texte.

N. B. Oui, mais par rapport à l'expérience de vie aussi, c'est tout à fait logique. Par exemple le fait d'employer le terme de afiction ».

M. v. S. C'est cohérent, tu veux dire.

N. B. Cohérent, oui.

M. v. S. Et logique?

N. B. Non, ça c'est autre chose. II y a cohérence parce que, pour moi, fiction - théorie, ça indique le désarroi dans lequel j'ai été durant deux ans: le plaisir et l'urgence toujours... c'est logique!

M. v. S. Si bien qu'il y a à la fois continuité et rupture. Rupture dans la mesure où cette fiction théorique mettra en scène des registres distincts les uns des autres et qui n'auront de rapports que dans leur distinction, dans leur hétérogénéité et, à la limite, dans leur incommunicabilité. Et continuité puisque cette expérience que tu es en train de formuler maintenant, elle était là, à l'œuvre, depuis le début dans tes premiers essais poétiques qui étaient des essais simples et aussi des essais théoriques?

J. F. Sans que tu le saches d'ailleurs.

N. B. Oui, sans que je le sache. La continuité, on ne la voit pas, mais elle est là, en fait, toujours.

M. v. S. C'est toujours un post facto, un après coup. La découverte de cette continuité participe de l'analyse au sens théorique, mais aussi de l'analyse au sens freudien.

N. B. A ce propos, il m'est venu une idée: une théorie, au contraire de l'image qu'on s'en fait habituellement en termes de science, de froideur, d'intellectualisation, serait liée très intimement à la fiction intérieure qui est celle de l'inconscient; d'où l'adhésion presque unanime aux théories d'Einstein, de Freud, du moins dans son temps, et de Marx. C'est tout simplement un «flash» que j'ai eu, mais j'y reviendrai dans des textes. 
M. v. S. Je crois personnellement que tu as raison, si l'on ajoute que c'est la rencontre historique qui assure ces paroles. C'est ce qui est arrivé à Diderot, Marx, Freud.

N. B. Oui, ça complète l'énonciation effectivement.

M.v.S. Ce n'est pas simplement la scène de l'inconscient, mais aussi l'autre scène, celle où tout se passe: la scène historique.

N. B. En fait, c'est la découverte du fonctionnement de notre inconscient à un moment précis de I'histoire, dans une histoire donnée.

octobre 1976

1. Un livre, roman, Montréal, Les Éditions du Jour, 1970.

2. Sold-out étreinte/illustration, roman, Montréal, Les Éditions du Jour, 1973.

3. French Kiss étreinte /exploration, roman, Montréal, Les Éditions du Jour, 1974.

4. Suite logique, poèmes, Montréal, Les Éditions de l'Hexagone, 1970.

5. Le Centre blanc, poèmes, Montréal, Les Éditions d'Orphée, 1970.

6. La Partie pour le tout, Montréal, Les Éditions de l'Aurore, 1975.

7. «E muet mutant ", daṇs la Barre du jour, $n^{\circ} 50$, Femme et langage, 1975, p. 10-27.

8. “Le Cortex exubérant ", dans la Barre du jour, $n^{\circ} 44,1974, p .2-22$.

9. Mordre en sa chair, poèmes, Les Éditions de l'Estérel, 1968.

10. La Nef des sorcières, pièce écrite en collaboration, Montréal, Les Éditions Quinze, 1976.

11. Inédit. 